\title{
CARACTERÍSTICAS DE PUBLICAÇÕES NACIONAIS SOBRE SÍNDROME DE BURNOUT
}

\author{
Jonathan Saidelles Corrêa ${ }^{1}$ \\ Luis Felipe Dias Lopes ${ }^{2}$ \\ Lucas Veiga Ávila ${ }^{3}$ \\ Lúcia dos Santos Albanio ${ }^{4}$ \\ Damiana Machado de Almeida ${ }^{5}$
}

\begin{abstract}
Resumo: A exaustão física e psicológica aliada ao estresse permanente no trabalho tende a conduzir ao desenvolvimento de doenças laborais, como a Síndrome de Burnout. Nesse contexto, estão inseridas principalmente aquelas profissões que presumem cuidados com pessoas, como enfermeiros, policiais, agentes penitenciários e docentes. Desse modo, o objetivo deste artigo é demonstrar o panorama das publicações sobre Burnout no contexto nacional na última década (2007-2016). Para isso, foram evidenciadas as principais características relacionadas ao tema, no que tange à área temática; distribuição de publicações por ano; autores e fontes. O método utilizado foi a bibliometria, com característica descritiva e abordagem quantitativa, utilizando como fonte de dados a Base SciELO - ScientificElectronic Library Online. Analisou-se um total de 169 publicações, constatando-se que a área de conhecimento de maior impacto foi a Enfermagem; o ano que mais ocorreu publicações foi 2014; o autor com maior número de registros foi Telma Ramos Trigo; a fonte mais relevante foi a Revista Latino-Americana de Enfermagem. Os resultados desta pesquisa possibilitam contribuir com o cenário acadêmico e empresarial, tendo em vista que auxiliam pesquisadores e gestores a compreenderem o comportamento do tema no cenário nacional.
\end{abstract}

Palavras-chave: Comportamento organizacional; Burnout; Estresse; Bibliometria.

\section{Characteristics of national publications on Burnout Syndrome}

\begin{abstract}
Physical and psychological exhaustion combined with permanent stress at work tends to lead to the development of occupational diseases, such as Burnout Syndrome. In this context, there are mainly those professions that presume to care for people, such as nurses, police officers, penitentiary agents and teachers. Thus, the aim of this article is to demonstrate the panorama of publications on Burnout in the national context in the last decade (2007-2016). For that, the main characteristics related to the theme were evidenced, in what concems: thematic area; distribution of publications per year; authors; and sources. The method used was bibliometry, with descriptive characteristic and quantitative approach, using
\end{abstract}

\footnotetext{
${ }^{1}$ Mestrando em Administração na Universidade Federal de Santa Maria.

${ }^{2}$ Professor Associado no Departamento de Ciências Administrativas da Universidade Federal de Santa Maria. Doutor em Engenharia de Produção pela Universidade Federal de Santa Catarina.

${ }^{3}$ Doutorando em Administração na Universidade Federal de Santa Maria - UFSM.

${ }^{4}$ Bacharel em Administração pela Faculdade Metodista de Santa Maria.

${ }^{5}$ Doutoranda em Administração na Universidade Federal de Santa Maria - UFSM.
} 
the SciELO Base - Scientific Electronic Library Online as the data source. A total of 169 publications were analyzed, confirming that: the area of knowledge with the greatest impact was Nursing; the year that most occurred publications was that of 2014; the author with the highest number of records was Telma Ramos Trigo; The most relevant source was the Revista Latino Americana de Enfermagem. The results of this research make it possible to contribute to the academic and business scenario, since they help researchers and managers to understand the behavior of the theme in the national scenario.

Keywods: Organizational behavior; Burnout; Stress; Bibliometry.

Resumen: El agotamiento físico y psicológico aliado al estrés permanente en el trabajo tiende a conducir al desarrollo de enfermedades laborales, como el Síndrome de Burnout. En este contexto, se insertan principalmente aquellas profesiones que presumen cuidados con personas, como enfermeros, policías, agentes penitenciarios y docentes. De este modo, el objetivo de este artículo es demostrar el panorama de las publicaciones sobre Burnout en el contexto nacional en la última década (2007-2016). Para ello, se evidenciaron las principales características relacionadas al tema, en lo que se refiere al área temática; Distribución de publicaciones al año; Autores y fuentes. El método utilizado fue la bibliometría, con característica descriptiva y enfoque cuantitativo, utilizando como fuente de datos la Base SciELO - ScientificElectronic Library Online. Se analizó un total de 169 publicaciones, constatando que el área de conocimiento de mayor impacto fue la Enfermería; El año que más ocurrió publicaciones fue 2014; El autor con mayor número de registros fue Telma Ramos Trigo; La fuente más relevante fue la Revista Latinoamericana de Enfermería. Los resultados de esta investigación posibilitan contribuir con el escenario académico y empresarial, teniendo en vista que auxilian a investigadores y gestores a comprender el comportamiento del tema en el escenario nacional.

Palabras-clave: Comportamiento organizacional; Quemadura; El estrés; Bibliometría.

\section{INTRODUÇÃO}

O contexto contemporâneo conduz à percepção que o trabalho vem ocupando um espaço maior na vida da maioria das pessoas. Pellegrini e Boer (2014) expõem que as pessoas estão frequentemente prolongando a sua exposição ao trabalho, à medida que optam por continuar laborando mesmo após a aposentadoria. De acordo com Camelo e Angerami (2008), a evolução tecnológica trouxe contribuições para o desenvolvimento do homem em seu contexto social, cultural e biológico; contudo, também, expôs a sua fragilidade física e emocional. Neste sentido, existem aspectos presentes na rotina de trabalho que interferem diretamente no desempenho individual de cada trabalhador.

Quando esses aspectos apresentam conotação negativa, eles podem acarretar prejuízos e problemas como o estresse ocupacional. Nessa perspectiva, está inserida a Síndrome de 
Burnout, a qual se refere à longa permanência do indivíduo em condições de insatisfação com as suas pretensões no ambiente laboral, motivando a exaustão física e mental. De acordo com Carvalho e Magalhães (2011, p. 204), “a Burnout geralmente leva à deterioração do bem-estar físico e emocional”.

Desse modo, evidencia-se que a não observância das condições de trabalho pode propiciar a ineficiência de inúmeras instituições, tanto no cenário público, como no privado, tendo em vista que os colaboradores são os principais responsáveis pelo alcance dos objetivos organizacionais. Controlar aspectos da rotina de trabalho relacionados à qualidade de vida, satisfação e bem-estar no trabalho são algumas entre as inúmeras abordagens capazes de ir de encontro à predisposição ao adoecimento de pessoas do contexto laboral.

Por ser um tema global, uma busca de anterioridade em Bases de dados contribui na análise de características relevantes de pesquisas já publicadas, haja vista a amplitude de conceitos e abordagens que tangenciam o tema. Desse modo, o objetivo deste estudo é demonstrar o panorama das publicações sobre a Síndrome de Burnout no contexto nacional na última década (2007-2016). Para isso, evidenciou-se as principais características das publicações, por intermédio da Base de Dados SciELO.

Convém salientar que a relevância deste estudo expressa-se tanto no contexto acadêmico como no empresarial. No cenário acadêmico, possibilita a orientação de pesquisadores sobre as principais características das publicações sobre Síndrome de Burnout. Já no âmbito empresarial, contribui para que gestores tenham acesso facilitado a fontes que possam abrigar casos práticos sobre o tema que, por ventura, possam auxiliar na tomada de decisão. Ademais, a identificação dos principais pesquisadores do tema pode induzir gestores a encontrar profissionais capazes de fornecerem consultoria empresarial.

\section{REFERENCIAL TEÓRICO}

A ausência de características benéficas no ambiente de trabalho pode comprometer a qualidade do ambiente laboral, conduzindo à ocorrência de situações prejudiciais aos colaboradores. Neste sentido, este tópico percorre o caminho desde os aspectos gerais sobre estresse até os relativos ao adoecimento organizacional. Além disso, evidencia a origem, principais conceituações e características da Síndrome de Burnout.

\subsection{Do estresse ao adoecimento organizacional}

A definição de estresse é proveniente do século XVII, porém, somente em 1926, um dos principais estudiosos do tema - Hans Selye - passou a descrevê-lo como um estado de 
tensão do organismo (LIPP, 2000). A banalização do conceito fez com que a sociedade empregue-o de maneira generalizada. Benevides-Pereira (2002) menciona que essa popularização contribuiu para que a expressão estresse fosse considerada sinônima de qualquer comportamento negativo incomum.

Embora haja esse emprego informal do conceito de estresse, no âmbito científico, a sua definição é controversa, uma vez que diversas áreas da ciência utilizam-no como objeto de estudo, principalmente as relacionadas às ciências biológicas e às ciências sociais e humanas. Em decorrência disso, Faro e Pereira (2013) afirmam que geralmente há ênfases distintas sobre o fenômeno na literatura, referindo-se, frequentemente, aos agentes estressores, à mediação cognitiva ou às consequências sobre a saúde.

O estresse é um estado em que o corpo emite reações a várias situações ambientais, físicas e sociais (FERRAZ; FRANCISCO; OLIVEIRA, 2014). Em outras palavras, quando afetada a homeostase do indivíduo, o seu organismo emite respostas, buscando restabelecer o seu equilíbrio interno.

O estresse pode ser considerado como mecanismo utilizado pelas pessoas para se adaptarem às exigências do meio externo ou para responderem a metas que fixam para si próprias. Características do meio externo podem estimular o indivíduo a se engajar em atividades cada vez mais complexas, desenvolvendo assim o seu potencial (TAMAYO, 2007, p. 25).

No entanto, nem todo estresse pode ser considerado prejudicial. Bianchi (2001) defende que o estresse também pode adotar conotação positiva, em que o indivíduo assume o evento estressante como um desafio. Sonnentag e Bayer (2005) também ratificam que nem toda carga de trabalho conduz ao estresse, mas aquela crônica e permanente na rotina de trabalho. De acordo com Bonez, Dal Moro e Sehnem (2013, p. 514), “é natural e até esperado que o ser humano experimente algum desconforto ocasional, que se assemelha aos sintomas de estresse".

Algum estresse é importante para a realização de qualquer atividade e que sua total ausência, assim como seu excesso, podem ser prejudiciais à saúde. Entretanto, o prolongamento de situações de estresse pode repercutir num quadro patológico, originando distúrbios transitórios ou mesmo doenças graves, como o estresse ocupacional (ABREU et al., 2002, p. 25).

Desse modo, cada pessoa considera o estresse sob um prisma distinto; enquanto para algumas configura um fator desmotivador, para outras, pode assumir conotação inversa. Em 
consonância com Kaur, Chodagiri e Reddi (2013) e Garbarino, Chiorri e Magnavita (2014) o estresse pode estar relacionado tanto à vida pessoal como à vida social dos indivíduos, manifestando-se de maneira distinta em cada pessoa.

Situações de estresse também podem ser verificadas no ambiente de trabalho. Benevides-Pereira (2002, p. 46) salienta que "o estresse pode acometer qualquer pessoa e, quando o agente desencadeador se refere à atividade desempenhada, o mais correto seria a designação de estresse ocupacional".O estresse ocupacional está diretamente relacionado ao esforço despendido na adaptação a ambientes inadequados, ou seja, ao desgaste físico e mental ocasionado pelo desempenho de atividades na presença de condições precárias (SANTOS; CUBAS, 2012; ZANELLI; ANDRADE; BASTOS, 2014).

Neste sentido, Alexopoulos et al. (2014) afirmam que os níveis de estresse têm efeito negativo profundo tanto na satisfação dos indivíduos no trabalho, como na qualidade de vida organizacional. Dependendo da intensidade dessas condições de desgaste, o estresse manifesta-se e acaba impactando diretamente a saúde das pessoas (WALVEKAR; AMBEKAR; DEVARANAVADAGI, 2015). Além de causarem a diminuição da produtividade individual de cada colaborador, esses componentes negativos podem ocasionar doenças de ordem física e psicológica. Os sintomas físicos e mentais de estresse tendem a manifestar-se tanto em nível individual como também no organizacional (PAIVAet al., 2013). Assim, é possível inferir que, por meio da interferência no desempenho individual das pessoas, o estresse ocupacional afeta o alcance dos objetivos das organizações.

Em determinados casos, a recorrência de situações de estresse ocupacional elevado propicia o desenvolvimento de problemas físicos e mentais mais graves. Isso está de acordo com os estudos de Monteiro, Dalagasperina e Quadros (2012, p. 20), os quais afirmam que “além do Estresse ocupacional, podem também aparecer outras doenças ocupacionais causadas pela recorrência e permanência do mesmo, entre as quais se destaca a Síndrome de Burnout". O tópico seguinte trata mais veementemente das principais características dessa doença.

\section{Síndrome de Burnout: origem, conceituações e características}

Em ambientes em que as condições de trabalho são precárias por longos períodos temporais, é alta a probabilidade do surgimento da Síndrome de Burnout. Essa doença tem transcendido a seara acadêmica, despertando também interesse de gestores (LEITER; BAKKER; MASLACH, 2014). Maslach, Schaufelli e Leiter (2001) defendem que o estresse 
crônico, típico do cotidiano do trabalho, a excessiva pressão, os conflitos e o baixo reconhecimento são fatores que influenciam na ocorrência da Síndrome de Burnout. Com pensamento congênere, Bakker e Demerouti (2013) afirmam que, em determinados casos, a disponibilização de recursos ofertada pelas organizações não é condizente com a quantidade de demandas de trabalho, contribuindo para a ocorrência de Burnout. Maslach e Jackson foram pioneiras ao estudar o tema, caracterizando-a como um problema proveniente do ambiente de trabalho e não oriundo do indivíduo (NEVES; OLIVEIRA; ALVES, 2014). No Quadro 1, estão relacionados alguns conceitos de diferentes autores sobre o tema.

Quadro 1 - Conceitos de Síndrome de Burnout

\begin{tabular}{|c|c|}
\hline Autor & Conceito \\
\hline Maslach e Jackson (1981) & \multirow{2}{*}{$\begin{array}{l}\text { Reação a uma tensão crônica que atinge profissionais de } \\
\text { serviço, principalmente aqueles voltados para atividades de } \\
\text { cuidado com outros, em que a oferta do cuidado ou serviço } \\
\text { frequentemente ocorre em situações de mudanças } \\
\text { emocionais. }\end{array}$} \\
\hline $\begin{array}{l}\text { Carvalho e Magalhães } \\
\qquad(2011)\end{array}$ & \\
\hline Benevides-Pereira (2002) & \multirow{2}{*}{$\begin{array}{l}\text { Consequência da influência prolongada de agentes } \\
\text { estressores sobre a rotina de trabalho de indivíduos. }\end{array}$} \\
\hline $\begin{array}{l}\text { Zanelli, Andrade e Bastos } \\
\text { (2014) }\end{array}$ & \\
\hline Gil-Monte (2005) & \multirow{2}{*}{$\begin{array}{l}\text { Uma resposta ao demasiado estresse laboral, principalmente } \\
\text { dos profissionais que trabalham com pessoas. }\end{array}$} \\
\hline $\begin{array}{l}\text { Gil-Monte, Carlotto e } \\
\text { Câmara (2010) }\end{array}$ & \\
\hline Lindblomet al. (2006) & $\begin{array}{l}\text { Relacionada a um processo de estresse no ambiente de } \\
\text { trabalho, proveniente da relação entre fatores psicossociais e } \\
\text { o trabalhador. }\end{array}$ \\
\hline
\end{tabular}

Fonte: Elaborado pelos autores.

Diferentemente do estresse, que pode apresentar aspectos positivos, a Síndrome de Burnout é sempre danosa à vida profissional e pessoal dos funcionários (BENEVIDESPEREIRA, 2002), visto que,, como já mencionavam Maslach e Jackson (1981), os trabalhadores sentem-se infelizes e insatisfeitos com a sua realização no trabalho. Em virtude disso, as organizações que buscam maior eficiência devem estar atentas ao atendimento, no mínimo, dos aspectos higiênicos do trabalho. De acordo com Benevides-Pereira (2002), os sintomas da Síndrome de Burnout podem ser tanto físicos, como comportamentais, psíquicos e defensivos.

Gil-Monte (2005) divide em dois perfis no processo de Burnout, sugerindo que há uma ordem de ocorrência dos sintomas. O primeiro perfil (primeiros sintomas) estaria ligado à danificação cognitiva e afetiva, que prejudicaria o modo como o colaborador desempenha a 
sua função; já o segundo perfil estaria relacionado às possíveis consequências dos primeiros sintomas, como sentimento de culpa por determinadas ações. Sob essa ótica, o autor considera a existência plena da síndrome somente na manifestação do segundo perfil, sendo o primeiro considerado apenas estresse. Depreende-se, assim, que a prevenção da Síndrome de Burnout está amparada na qualidade de vida no trabalho.

Por outro lado, é relevante salientar que a identificação de sintomas de Burnout em um funcionário de determinada instituição não significa a presença nos demais integrantes, isso porque o progresso dessa doença ocorre de forma singular em cada indivíduo, podendo os seus efeitos levarem anos para serem percebidos (RUDOW, 1999). Em virtude disso, Maslach (2003) afirma que as estratégias não devem ser direcionadas para os indivíduos, mas diretamente no ambiente de trabalho das organizações, de maneira preventiva.

Logo, é possível inferir que a estratégia ideal é aquela implementada anteriormente à manifestação da síndrome, com foco nas rotinas de trabalhos, proporcionando que os indivíduos desempenhem as suas atividades de maneira satisfatória. Caso essa prevenção não ocorra de maneira tempestiva, as ações corretivas deverão ser direcionadas aos indivíduos, pois cada um reagirá de forma distinta. Exposta a revisão de literatura, o tópico 3 relata o caminho metodológico adotado para atingir o objetivo inicialmente proposto.

\section{Método}

A presente pesquisa configura-se como um estudo bibliométrico descritivo, com abordagem quantitativa, o qual visa alcançar um aprofundamento sobre as publicações relevantes presentes na literatura nacional sobre o tema Síndrome de Burnout. Para Pritchard (1969), a bibliometria é aplicada em pesquisas que visam analisar estatisticamente os processos de comunicação escrita. De modo semelhante, Silva (2004) e Leite Filho (2008) mencionam que ela objetiva a avaliação da atividade científica ou técnica de um determinado campo do conhecimento através do estudo quantitativo de publicações.

No campo das Ciências Sociais, a bibliometria tem a função recorrente de averiguar a produção de artigos em determinadas áreas, mapear as comunidades acadêmicas e identificar as redes de pesquisadores e suas motivações (NEDERHOF, 2006; CHUEKE; AMATUCCI, 2015).Esses estudos estatísticos que analisam as características de publicações de maneira longitudinal costumam ser balizados por três leis: Lei de Lotka, Lei de Brandford e Lei de Zipf.

A Lei de Lotka (lei do Quadrado Inverso) propõe que determinado número de pesquisadores produz muito em determinada área - e são mais citados -, sendo que um 
grande número de pesquisadores produz pouco - e são menos citados (ÁVILA et al., 2014; MACHADO JUNIOR, et al., 2016). Em outros termos, transfere-se parcela da relevância do artigo ao autor responsável por sua publicação, principalmente para aqueles reconhecidos na academia por possuírem elevada produção científica em determinados temas.

Por sua vez, a lei de Bradford (lei de Dispersão) estima a importância de periódicos que atuam nas mais variadas áreas do conhecimento. O objetivo dessa lei, na perspectiva de Araujo (2006), é evidenciar a disposição dos artigos nos periódicos, no intuito de determinar aqueles mais relevantes para determinados assuntos. Assim, no instante que um periódico dispõe-se a publicar um assunto considerado novo, há uma probabilidade de que outros autores que estudam o tema também procurem encaminhar as suas produções para o mesmo periódico. Com esse movimento, há uma especialização na revista sobre o tema devido às recorrentes publicações relevantes.

Por fim, a lei de Zipf (lei do Mínimo Esforço) "relaciona a frequência de palavras e o significado das mesmas para a área de pesquisa" (QUEVEDO-SILVA et al., 2016, p. 249). Neste caso, a quantidade de vezes que uma palavra é mencionada por autores define os principais temas abordados em distintas ciências. Tal lei está relacionada à enumeração dos tópicos mais relevantes sobre determinados temas (hot topics).

Convém salientar que o presente estudo bibliométrico auferiu resultados relativos a duas das três leis bibliométricas. Os resultados referente à Lei de Zipf não foram possíveis em virtude da Base de Dados SciELO não permitir o cálculo dos índices h e m para identificação dos hot topics sobre o tema. Desse modo, optou-se pela análise bibliométrica sobre Síndrome de Burnout, englobando publicações de 2007 a 2016 contidas na base de dados SciELO. Exposto o caminho bibliométrico, na sequência, serão evidenciadas as principais características das publicações sobre o tema Síndrome de Burnout.

\section{Análise dos Dados}

Nesta seção, estão demonstrados os principais resultados relativos ao estudo bibliométrico nacional realizado sobre o tema na última década. Foram analisadas as dez primeiras posições classificadas por maior número de registros (área temática, publicações por ano e fonte) e o maior número de citações do artigo (autores). Por meio da inserção do descritor "Burnout" no sistema de busca da base de dados SciELO, destacaram-se 169 publicações realizadas no Brasil no período de 2007 a 2016.

A primeira característica do estudo bibliométrico refere-se às áreas temáticas relacionadas ao tema. Neste sentido, restou evidenciado que a área de pesquisa que engloba o 
maior número de publicação é a Enfermagem, com 38 registros. Também com quantitativo expressivo, destacaram-se as áreas de Saúde pública, ambiental e ocupacional (31) e Psicologia multidisciplinar (20). Logo após estão as áreas de Física multidisciplinar (13), Ciências e serviços da saúde (11), Medicina geral e interna (10), Sociologia (10), Educação e pesquisa educacional (8), Psiquiatria (7) e Psicologia educacional (7).

Para verificar o panorama da produção científica ao longo dos últimos dez anos, demonstrou-se também a distribuição do quantitativo de publicações nacionais em cada período na Imagem1.

Imagem1 - Publicações por ano sobre Síndrome de Burnout

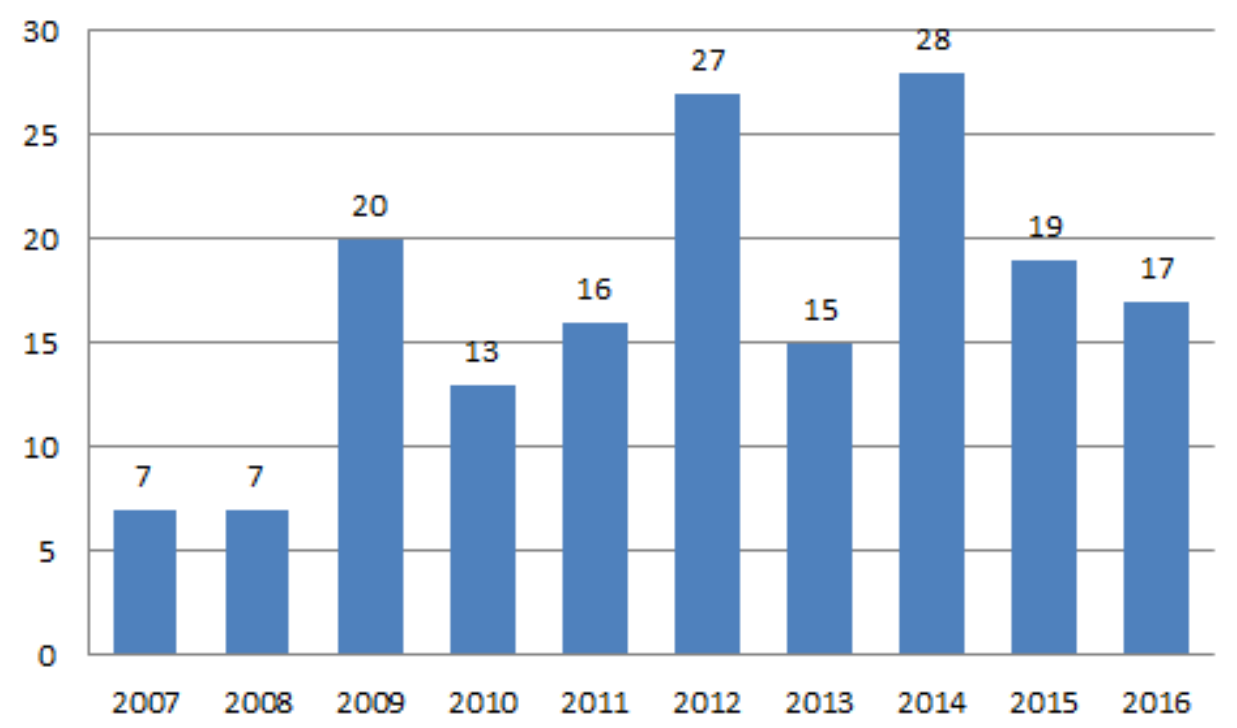

Fonte: Scielo (2017).

Ao analisar os dados da Imagem 1, percebe-se uma oscilação no interesse dos pesquisadores sobre o tema ao longo da última década. Nos anos de 2007 e 2008, a quantidade de publicações foi a menos significativa, com sete em cada um. Por sua vez, um aumento expressivo nas publicações ocorre no ano de 2009, subindo para 20 a quantidade de produção anual. Os anos mais significativos foram 2012 e 2014, com 27 e 28 publicações, respectivamente. $\mathrm{O}$ ano de 2016 foi o $5^{\circ}$ com maior número de publicações anuais. Conforme já frisado anteriormente, a demonstração do panorama das publicações de modo longitudinal é relevante para que se demonstre o estágio de significância quanto à contemporaneidade do tema.

Também se torna relevante evidenciar os principais autores que publicam sobre o assunto, no intuito de atender os requisitos de uma das três leis da Bibliometria, a Lei de 
Lotka. Nessa ótica, verificou-se que a autora do estudo mais citado é Telma Ramos Trigo, com 28 citações. Junto com essa autora, outros três completam as quatro primeiras posições: Davi de Souza (23), Fernanda Meneghini (18) e Mary Sandra Carlotto (18). Após esses autores, figuram Flávia Dutra (14), Dalton de Souza Barros (14), Denise Albieri Jodas (13), Letícia de Lima Trindade (11), Rosângela Marion da Silva (10) e Luciano Garcia Lourenção (10).

Outra informação significativa que satisfaz a lei bibliométrica de Bradford é a relação das principais fontes em que estão depositados os escritos sobre Burnout. Assim, a fonte que apresentou o maior número de produções em relação ao total de registros foi a Revista LatinoAmericana de Enfermagem (11), a qual é o canal oficial de divulgação científica da Escola de Enfermagem de Ribeirão Preto da Universidade de São Paulo e do Centro Colaborador da OPS/OMS para o Desenvolvimento da Pesquisa em Enfermagem. Em seguida estão: Revista da Escola de Enfermagem da USP (10), Acta Paulista de Enfermagem (9), Psicologia: Reflexão e Crítica (9), Revista Brasileira de Educação Médica (9), Ciência \& Saúde Coletiva (8), Revista de Saúde Pública (6), Psicologia Escolar e Educacional (5), Cadernos Saúde Coletiva (4) e Psico-USF (4).

Expostas essas características, ficam atendidas duas leis bibliométricas referentes à identificação dos principais autores (Lei de Lotka) e das principais fontes (Lei de Bradford). A última lei - Lei de Zipf - não foi retratada neste estudo em virtude da base de dados empregada não possuir ferramentas bibliométricas para cálculo de índice h e m. Além de evidenciar características relativas às leis supracitadas, salientou-se também as principais áreas de pesquisa e a quantidade de publicações por ano na década pesquisada.

\section{Considerações Finais}

O intuito desta pesquisa foi demonstrar o panorama das publicações sobre Síndrome de Burnout nacionalmente na última década (2007-2016), por intermédio da base de dados SciELO. Inicialmente, a análise permitiu concluir que, no geral, o número de publicações sobre o tema apresentou um comportamento oscilante no período, totalizando 169 publicações.

Algumas características sobre o tema merecem maior destaque com base nos resultados obtidos nas primeiras posições, pois ensejam o estabelecimento de algumas relações de semelhança e reflexões. No que tange à área temática, percebe-se que as ciências que possuem finalidade de cuidados com a saúde são as principais fomentadoras de publicações sobre Síndrome de Burnout, embora haja considerável interdisciplinaridade. 
Quanto à autora com maior número de registros, figurou Telma Ramos Trigo, a qual é Médica psiquiatra colaboradora do grupo de pesquisas do Interconsultas - Instituto de Psiquiatria do Hospital das Clínicas da Faculdade de Medicina da Universidade de São Paulo, sendo que ela possui mestrado com ênfase em Síndrome de Burnout ou Esgotamento Profissional. Por sua vez, a principal fonte também vai ao encontro das características das áreas temáticas e do principal autor, pois publica resultados de pesquisa científica em enfermagem e outras áreas de interesse para profissionais de saúde.

Com esta exposição, espera-se contribuir tanto para o contexto acadêmico como para o empresarial, dada a importância do panorama construído sobre o tema. Como limitação do estudo, destaca-se a utilização de apenas uma base de dados específica, de maneira que ela não possibilitou a identificação dos hottopics. Por essa razão, sugere-se que estudos futuros dessa natureza alcancem uma maior amplitude, abrangendo outras bases de dados e também eventos acadêmicos de impacto científico.

\section{Referências}

ABREU, Klayne L. et al. Estresse ocupacional e Síndrome de Burnout no exercício profissional da psicologia.Psicologia: Ciência e Profissão, v. 22, n.2, Brasília. 2002.

ALEXOPOULOS, Evangelos C. et al. Exploring Stress Levels, Job Satisfaction, and Quality of Life in a Sample of Police Officers in Greece. Safetyand Health atWork, v. 5, n. 4, p. 210215. 2014.

ARAÚJO, Carlos A. A. Bibliometria: evolução histórica e questões atuais. Em Questão, v. 12, n. 1, p. 11-32. 2006.

ÁVILA, Lucas V. et al. Características das publicações sobre Empreendedorismo (Social) no Web of Science no período 2002-2011. Administração Pública e Gestão Social, v. 6, n.2, p. 88-100. 2014.

BAKKER, Arnold B.; DEMEROUTI, Evangelia. La teoría de las demandas y los recursos laborales. JournalofWorkandOrganizationalPsychology, v. 29, p. 107-115. 2013.

BENEVIDES-PEREIRA, Ana M. T. B (Org.). Burnout: quando o trabalho ameaça o bemestar do trabalhador. 3. ed.- São Paulo: Casa do Psicólogo. 2002.

BIANCHI, Estela R. F. Conceito de Stress: evolução histórica. Nursing, v. 4, n. 39, p. 16-19. 2001. 
BONEZ, Aline; DAL MORO, Elisamara; SEHNEM, Scheila Beatriz. Saúde mental de agentes penitenciários de um presídio. Psicol. Argum., Curitiba, v. 31, n. 74, p. 507-517, jul./set. 2013.

CAMELO, S. H. H; ANGERAMI, Emília L. S. Riscos psicossociais no Trabalho que podem levar ao estresse: uma análise da literatura. Revista Ciência, Cuidado e Saúde, v. 7, n. 2, p. 232-240, abr./jun. 2008.

CARVALHO, C. G.; MAGALHÃES, S. R. Síndrome de Burnout e suas consequências nos profissionais de enfermagem. Revista da Universidade Vale do Rio Verde, Três Corações, v. 9, n. 1, p. 200-210, jan./jul. 2011.

CHUEKE, G. V.; AMATUCCI, M. O que é bibliometria? Uma introdução ao Fórum. Revista Eletrônica de Negócios Internacionais, v.10, n. 2, p. 1-5, São Paulo, maio/ago. 2015.

FARO, André; PEREIRA, Marcos E. Medidas do estresse: uma revisão narrativa. Psicologia, Saúde \& Doenças, v. 14, n. 1, p. 101-124. 2013.

FERRAZ, Flávio C; FRANCISCO, Fernando R.; OLIVEIRA, Celso S. Estresse no ambiente de trabalho. Archives of Health Investigation, v. 3, n. 5, p. 1-8. 2014.

GARBARINO, Sergio; CHIORRI, Carlo; MAGNAVITA, Nicola. Personality traits of the Five-Factor Model are associated with work-related stress in special force police officers. International Archives of Occupational and Environmental Health, v. 87, n. 3, p. 295-306. 2014.

GIL-MONTE, Pedro R.El síndrome de quemarse por eltrabajo (Burnout): una enfermedad laboral enlasociedaddelbienestar. Madrid: Pirámide. 2005.

GIL-MONTE, Pedro R.; CARLOTTO, Mary S.; CÂMARA, Sheila G. Validação da versão brasileira do "Cuestionario para laEvaluacióndel Síndrome de Quemarse por elTrabajo" em professores. Revista SaúdePública, v. 44, n. 1, p.140-7. 2010.

KAUR, Ravneet; CHODAGIRI, Vamsi K.; REDDI, Narasimha K.A Psychological Study of Stress, Personality and Coping in Police Personnel.IndianJournalofPsychological Medicine, v. 35, n. 2, p. 141-147. 2013.

LEITE FILHO, G. A. Padrões de produtividade de autores em periódicos e congressos na área de contabilidade no Brasil: um estudo bibliométrico. Revista de Administração Contemporânea, Curitiba, v. 12, n. 2, p. 533-554, abr./jun. 2008.

LEITER, Michael P.; BAKKER, Arnold B.; MASLACH, Cristina.Burnout at work. New York: Taylor \& Francis. 2014.

LINDBLOM, Karin M. et al. Burnout in the working population: Relations to psychosocial work factors. International Journal of Behavioral Medicine, v. 13, n. 1, p. 51-59. 2006. 
LIPP, Marild E. N. O Stress está dentro de você. 2. ed. São Paulo: Contexto. 2000.

MACHADO JUNIOR, Celso et al. As Leis da Bibliometria em Diferentes Bases de Dados Científicos. Revista de Ciências da Administração, v. 18, n. 44, p. 111-123. 2016.

MASLACH Cristina. Job Burnout: new directions in research and intervention. Current Directions in Psychological Science, v. 12, n. 5, p. 189-92. 2003.

MASLACH Cristina; JACKSON, Susan E.The measurement of experienced Burnout.Journal of Occupational Behaviour, v. 2, p. 99-113. 1981.

MASLACH, Cristina; SCHAUFELLI, Wilmar B.; LEITER, Michael P. Job Burnout.Annual Review of Psychology. v. 52, n. 1, p. 397-422. 2001.

MONTEIRO, J. K.; DALAGASPERINA, P.; QUADROS, M. O. Professores no limite: o estresse no trabalho do ensino privado no Rio Grande do Sul. Porto Alegre: Cartaeditora. 2012.

NEDERHOF, A. J. Bibliometric monitoring of research performance in the social sciences and the humanities: A review. Scientometrics, v. 66, n. 1, p. 81-100. 2006.

NEVES, V. F.; OLIVEIRA, Á. F.; ALVES, P. C. Síndrome de Burnout: Impacto da Satisfação no Trabalho e da Percepção de Suporte Organizacional. Psico, Porto Alegre, PUCRS, v. 45, n. 1, p. 45-54, jan.-mar. 2014.

PAIVA, Kely C. M. et al. Estresse Ocupacional e Burnout de jovens trabalhadores. In Anais...Rio de Janeiro: XXXVII EnANPAD, p. 1-16. 2013.

PELlEGRINI, B. M. de.; BOER, N. O Sentido do Trabalho: A Visão Humanista e a Aposentadoria. Saber Humano, v. 4, n. 5. 2014.

PRITCHARD, A. Statistical bibliography or bibliometrics?Journal of Documentation, v. 25, n. 4, p. 348-349, dec. 1969.

QUEVEDO-SILVA, Filipe et al. Estudo bibliométrico: orientações sobre sua aplicação. Revista Brasileira de Marketing - ReMark, v. 15, n. 2. 2016.

RUDOW, Bernd. Stress and Burnout in the teaching profession: european studies, issues, and research perspectives. Cambridge: Cambridge University Press. 1999.

SANTOS, Alvaro S.; CUBAS, Marcia R. Saúde coletiva: linhas de cuidado e consulta em enfermagem. Rio de Janeiro: Elsevier. 2012. 
SILVA, Márcia R. Análise bibliométrica da produção científica docente do programa de pósgraduação em educação especial/UFSCar: 1998-2003. Dissertação (Mestrado em Educação Especial). Universidade Federal de São Carlos. 2004.

SONNENTAG, Sabine; BAYER, Ute-Vera.Switching off mentally: Predictors and consequences of psychological detachment from work during off-job time.JournalofOccupational Health Psychology, v. 10, p. 393-414. 2005.

TAMAYO, Alvaro. Impacto dos Valores da Organização sobre o Estresse Ocupacional. Revista de Administração Contemporânea, v. 1, n. 2, p. 20-33. 2007.

WALVEKAR, Snjeev S; AMBEKAR, Jeevan G.; DEVARANAVADAGI, Basavaraj B. StudyonSerum Cortisol andPerceived Stress Scale in the Police Constables. JournalofClinicalandDiagnosticResearch, v. 9, n. 2, p. 10-14. 2015.

ZANELLI, José C. ANDRADE, Jairo E. B.; BASTOS, Antônio V. B. Psicologia, organizações e trabalho no Brasil. 2. ed. Dados eletrônicos. Porto Alegre: Artmed. 2014. 